\title{
Number of Daily Doses Does Not Affect Compliance with Flucloxacillin Prescriptions
}

\author{
Elisabeth Rönnbäck ${ }^{1}$, Sofia Kälvemark Sporrong ${ }^{1,2^{*}}$, Anders Österlund ${ }^{3}$ \\ ${ }^{1}$ Department of Pharmacy, Uppsala University, Uppsala, Sweden; ${ }^{2}$ Department of Pharmacy, University of Copenhagen, Copenhagen, \\ Denmark; ${ }^{3}$ Department of Communicable Disease Control and Prevention, Norrbotten County Council, Luleå, Sweden. \\ Email: *sofia.sporrong@sund.ku.dk
}

Received July $5^{\text {th }}, 2013$; revised August $6^{\text {th }}, 2013$; accepted August $16^{\text {th }}, 2013$

Copyright ( 2013 Elisabeth Rönnbäck et al. This is an open access article distributed under the Creative Commons Attribution License, which permits unrestricted use, distribution, and reproduction in any medium, provided the original work is properly cited.

\begin{abstract}
Objective: To achieve optimal effect with beta-lactam antibiotics, regimens with frequent dosages have been found necessary. However, if compliance is negatively influenced by more frequent dosages, this might hamper the effect of the treatments. Therefore, we have studied whether the numbers of daily dosages influence the compliance with prescribed flucloxacillin regimens. Design: A prospective interview study of patients with different dosing regimens of flucloxacillin. Setting: In the study, 200 patients with different dosing regimens of flucloxacillin underwent structured telephone interviews in order to reveal their compliance with the medication. Of these, 13 were prescribed twice-daily doses, 163 three times daily and 24 patients four times daily regimens. Results: There were no statistically significant differences between missed doses and prescribed numbers of daily doses. There was, however, a significant difference in the number of daily doses and experiences associated with the medication. Conclusions: This study shows that patients comply well with regimens of up to four daily doses of flucloxacillin, despite experiencing increasing difficulties with regimens of more frequent dosages.
\end{abstract}

Keywords: Antibiotics; Compliance; Flucloxacillin; Treatment; Sweden

\section{Introduction}

Compliance with antibiotic regimens is important, not only to get optimal effect from the drug, but also to prevent bacterial resistance [1]. Non-compliance can be intentional or unintentional; the latter can for example be due to forgetfulness or shortcomings in communication between patient and health care provider [2]. Lack of compliance has been identified as one contributor to unsuccessful antibiotics therapies, predominantly due to forgetfulness, adverse effects and patients determining themselves that they have been cured [3-5].

Earlier studies have shown compliance with antibiotics to be connected with dosing, with better adherence to regimens of fewer doses per day [6-8]. On the other hand, it is well known that for beta-lactam antibiotics, regimens with more frequent dosages have been found necessary to reach blood concentrations in sufficient time above MIC (minimum inhibitory concentration) to achieve optimal effect $[9,10]$.

Norrbotten County Council, responsible for healthcare

\footnotetext{
"Corresponding author.
}

in the northernmost part of Sweden, has since 2011 recommended four daily doses of beta-lactam antibiotics in order to optimize efficacy. However, this could influence the compliance and thus the recommendation could be counteractive. Thus, this study was done to compare patient experiences of different prescribed doses of flucloxacillin, and to reveal whether bad compliance with more frequent dosages could hamper the effect of the treatments.

\section{Material and Methods}

All patients who obtained flucloxacillin during the study time, 1st of April - 15th of May and 1st of September 15th of October in 2011, at five pharmacies in the county of Norrbotten, Sweden, were asked by pharmacy staff to participate in the study. The five largest pharmacies in the county, responsible for dispensing $64 \%$ of all flucloxacillin prescriptions in 2010, were enrolled in the study. Inclusion criteria for patients were: fluency in Swedish, collecting a prescription for oneself or one's own children, and written consent to an interview about the treatment after the course had ended. Two patients 
who terminated their course after one and three days respectively were excluded as they were viewed as not having had enough experience. The patients that were enrolled in the study were not informed about that the main purpose of the interview was to collect data concerning compliance to the prescribed doses. Structured telephone interviews were conducted with the patient a few days after estimated end of course. The questions were predominantly retrospective. The choice of method, i.e. telephone interviews, was to try to counteract any memory bias that might occur, as the respondent can expand on answers, e.g. communicate that they are uncertain, and the interviewer can prompt answers to get as correct data as possible [11].

Questions included whether the respondent had not taken the medicine any day; if so why, and how the respondent experienced taking the medicine several times per day.

The Statistical Package for Social Sciences (SPSS) was used for the statistical analysis. Answers to openended question were collected into broad categories.

To get informed consent, patients were given written information and if they accepted they were to give the pharmacy staff their phone numbers, as well as preferred times for interviews. The interviewer also collected information from the prescription, including age, sex, date of dispensing, dosage, package size, diagnosis and length of course.

Ethical approval was applied for at the regional ethical review board in Uppsala, which stated that no ethical approval was needed, but all the same declared no ethical obstacles.

\section{Results}

In total 325 patients (160 women and 165 men) were asked to participate in the study. Of those, 230 patients (112 women and 118 men) accepted. The interviewer finally succeeded in contacting 200 patients, 99 women (mean age 49 years) and 101 men (mean age 51 years).

Flucloxacillin was mainly prescribed for the treatment or prevention of skin and soft tissue infections (see Table 1). Three different dosage regimens were used; twice, three times, and four times daily. Three times daily was the predominant regimen used (see Table 2). Despite the somewhat small sample, it was evident that the self-estimated compliance with the different dosage regimens did not differ significantly (see Figure 1). However, it was just as clearly shown that the patients found it gradually more problematic to take more daily doses (see Figure 2).

Out of the 200 patients 58 (29\%) reported that they had missed doses. Among these 58 patients 25 (43\%) had forgotten only one or two doses. Forgetfulness was the main reason for missing doses; 36 (77\%) had missed as a
Table 1. Indications for flucloxacillin treatments.

\begin{tabular}{cc}
\hline Indication & No of Patients (proportion) \\
\hline Skin or soft tissue infections & $155(78 \%)$ \\
Prophylaxis after minor surgery & $24(12 \%)$ \\
Bone or joint infections & $9(5 \%)$ \\
Other infections & $5(3 \%)$ \\
Unknown & $7(4 \%)$ \\
\hline
\end{tabular}

Table 2. Prescribed doses of flucloxacillin to interviewed study patients.

\begin{tabular}{cc}
\hline Prescribed dose of flucloxacillin & No of Patients (proportion) \\
\hline Twice daily & $13(6.5 \%)$ \\
Three times daily & $163(82 \%)$ \\
Four times daily & $24(12 \%)$ \\
\hline
\end{tabular}

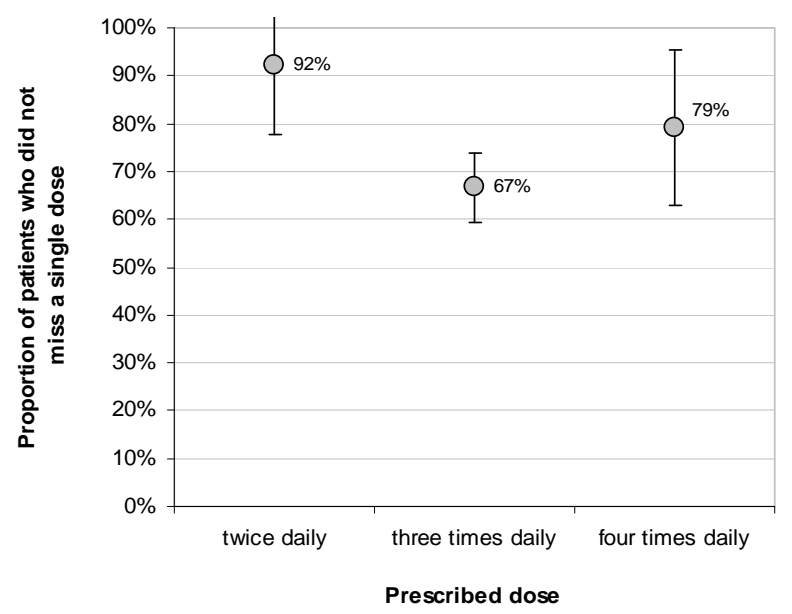

Figure 1. Proportion of patients who reported that they did not miss a single dose. Bars showing $95 \%$ confidence intervals.

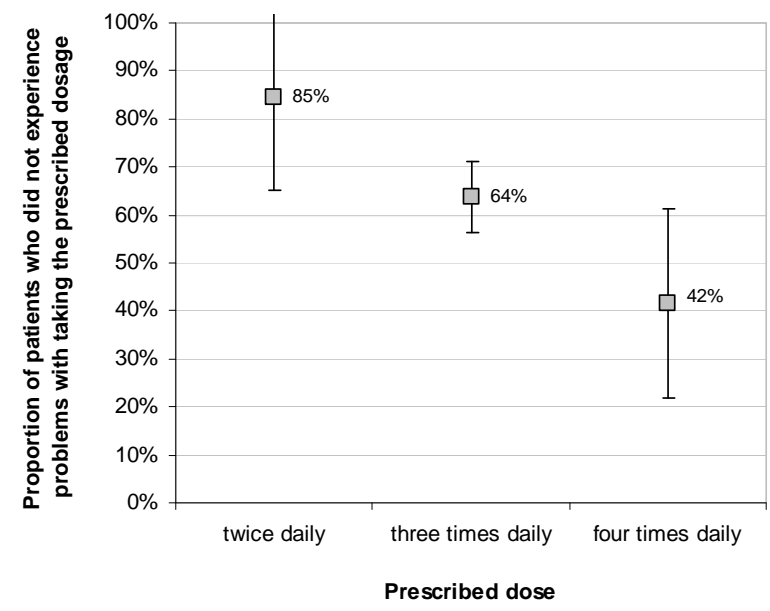

Figure 2. Proportion of patients who reported that they did not experience any problems with taking the prescribed dosage of antibiotics. Bars showing $95 \%$ confidence intervals. 
result of that. Missing doses was mainly due to changes in daily routines, such as going back to work. Adverse events were reported from three (6\%) patients (see Table 3).

\section{Discussion}

During the past decade, there has been an increasing awareness of adhering to pharmacodynamic principles [9] in antibiotic treatments. Thus, the importance of doses being administered more frequently for the time-dependent antibiotics, such as the beta-lactams, has become evident [10]. The finding that more frequent daily dosing has led to poor compliance with the prescriptions $[3,11]$ has thus been thought to be an obstacle to optimal antibiotic dosing. However, recently Eide and co-workers published data from a study on antibiotic treatment in primary health care in Norway which showed no significant differences in compliance between antibiotic regimens of three and four daily doses [12]. The results of our study further strengthen their findings that it is not evident that more doses lead to poorer compliance. Furthermore, our data show no differences in compliance to dosage regimens of twice, three times, or four times daily, despite reports from the patients that they find it increasingly difficult with more daily doses.

However, our data show that patients seem to find it more problematic to take more doses per day. As flucloxacillin is often used under a short period of time, this might be an obstacle that is fairly easily overcome. Communication between prescriber and patient, and pharmacy staff and patient, in order to encourage patients to put up with these inconveniences should be highlighted.

The results of this study are based on the answers from patients during interviews. To reduce the risk of interviewer's bias, all interviews are carried out in a systematic way by the same interviewer. However, there is still a risk that missed doses are under-reported in the interviews. Furthermore, only 200 out of the 325 patients (62\%) that get flucloxacillin dispensed at the pharmacies participate in the study. Despite the risk of under-reporting and a slight selection, there are no reasons to believe that patients are more prone to be biased in respect to certain dosing regimens. As this is a real life study, differences in number of patients per regimen are inevitable.

These results might reflect the intensive focus on antibiotic consumption and resistant bacteria in Swedish media during the past year. As a consequence, patients may have become more aware that unnecessary consumption of antibiotics might be harmful, and that compliance with the prescribed dosages is important for maximal effect in those circumstances where antibiotics are needed. Also, prescribers and pharmacy staff might have been more prone to motivate patients with four doses, as this regimen
Table 3. Answers to the question of "What was the reason for missing the dose/doses?”

\begin{tabular}{cc}
\hline Reason & No of Answers (proportion) \\
\hline I forgot & $36(77 \%)$ \\
I ran out of medicine & $2(4 \%)$ \\
Child with difficulties taking the & $2(4 \%)$ \\
medicine & $4(9 \%)$ \\
Inconvenient due to activities & $3(6 \%)$ \\
Adverse reaction & 3 \\
\hline
\end{tabular}

is fairly new to them as well.

\section{Acknowledgements}

We thank the staff at the Apoteket $A B$ pharmacies in Norrbotten, Sweden, including Blåklockan in Sunderbyn, Manhem in Kalix, Lejonet in Luleå, Laxen in Boden, and Renen in Piteå. The project was performed with a grant from Norrbotten County Council, Sweden.

\section{REFERENCES}

[1] WHO, "The Evolving Threat of Antimicrobial Resistance: Options for Action,” WHO Library Cataloguing-in-Publication Data, 2012.

http://whqlibdoc.who.int/publications/2012/97892415031 81_eng.pdf

[2] K. Krigsman, "Refill Adherence to Long-Term Drug Treatment with a Focus on Asthma/COPD Medication,” Ph.D. Thesis, Uppsala University, 2007.

[3] J. Ho, D. M. Taylor, M. S. Cabalag, A. Ugoni and M. Yeoh, "Factors That Impact on Emergency Department Patient Compliance with Antibiotic Regimens," Emergency Medicine Journal, Vol. 27, No. 11, 2010, pp. 815820. doi:10.1136/emj.2009.081984

[4] J. W. Cals, R. M. Hopstaken, P. H. Le Doux, G. A. Driessen, P. J. Nelemans and G. J. Dinant, "Dose Timing and Patient Compliance with Two Antibiotic Treatment Regimens for Lower Respiratory Tract Infections in Primary Care,” International Journal of Antimicrobial Agents, Vol. 31, No. 6, 2008, pp. 531-536. doi:10.1016/j.ijantimicag.2008.01.029

[5] B. S. Aronson, "Antibiotic-Taking Experiences of Undergraduate College Students," Journal of the American Academy of Nurse Practitioners, Vol. 18, No. 12, 2006, pp. 591-508. doi:10.1111/j.1745-7599.2006.00184.X

[6] K. Przemyslaw, "Comparison of Patients' Compliance with Once-Daily and Twice-Daily Antibiotic Regimens in Respiratory Tract Infections: Results of a Randomized Trial,” Journal of Antimicrobial Chemotherapy, Vol. 59, No. 3, 2007, pp. 531-536.doi:10.1093/jac/dkl528

[7] S. Hernández, C. Bayona, M. Hernández, A. Moragas and O. Calvio, "Compliance Rate of Antibiotic Therapy in Patients with Acute Pharyngitis Is Very Low, Mainly When Thrice-Daily Antibiotics Are Given," Revista Espanola De Quimioterapia, Vol. 22, 2009, pp. 20-24. 
[8] J. Claxton, J. Cramer and C. Pierce, “A Systematic Review of the Associations between Dose Regimens and Medication Compliance,” Clinical Therapeutics, Vol. 23, No. 8, 2001, pp. 1296-1310. doi:10.1016/S0149-2918(01)80109-0

[9] W. A. Craig, "Choosing an Antibiotic on the Basis of Pharmacodynamics,” Ear, Nose \& Throat Journal, Vol. 77, No. 6, 1998, pp. 7-11.

[10] C. A. DeRyke, S. Y. Lee, J. L. Kuti and D. P. Nicolau, "Optimising Dosing Strategies of Antibacterials Utilising Pharmacodynamic Principles: Impact on the Development of Resistance,” Drugs, Vol. 66, No. 1, 2006, pp. 1-

\section{4. doi:10.2165/00003495-200666010-00001}

[11] C. Llor, N. Sierra, S. Hernández, A. Moragas, M. Hernández, C. Bayona and M. Miravitlles, "The Higher the Number of Daily Doses of Antibiotic Treatment in Lower Respiratory Tract Infection, the Worse the Compliance," Journal of Antimicrobial Chemotherapy, Vol. 63, No. 2, 2009, pp. 396-399. doi:10.1093/jac/dkn472

[12] T. B. Eide, V. C. Hippe and M. Brekke, “The Feasibility of Antibiotic Dosing Four Times per Day: A Prospective Observational Study in Primary Health Care,” Scandinavian Journal of Primary Health Care, Vol. 30, No. 1, 2012, pp. 16-20. doi:10.3109/02813432.2012.654196 